\title{
¿Qué tipos de métodos de extensión prefieren y usan los extensionistas rurales? Comparación internacional y construcción de una tipología de acuerdo a sus métodos
}

\author{
What extension methods do rural extension agents prefer and use? \\ International comparison and construction of a typology according to \\ their methods \\ Fernando Pablo Landini (1), Felipe Gallardo-López² (1) \\ ${ }^{1}$ Consejo Nacional de Investigaciones Científicas y Técnicas (CONICET) e Instituto de Investigaciones Científicas, Universidad de la \\ Cuenca del Plata (UCP), Posadas (Misiones), Argentina. E-mail: landini_fer@hotmail.com \\ ${ }^{2}$ Programa de Postgrado en Agroecosistemas Tropicales, Colegio de Postgraduados (CP), Campus Veracruz, Veracruz, México. \\ E-mail: felipeg|@colpos.mx
}

\begin{abstract}
Cómo citar: Landini, F. P., \& Gallardo-López, F. (2022). ¿Qué tipos de métodos de extensión prefieren y usan los extensionistas rurales? Comparación internacional y construcción de una tipología de acuerdo a sus métodos. Revista de Economia e Sociologia Rural, 60(3), e238534. https://doi.org/10.1590/1806-9479.2021.238534
\end{abstract}

Resumen: En contraste con el amplio corpus de literatura orientado a discutir diferentes enfoques o filosofías de extensión, existe un limitado interés por analizar los métodos de extensión rural. Este estudio cuantitativo analiza los tipos de métodos que prefieren y usan extensionistas rurales de nueve países, y explora la relación entre tipos de métodos y características sociodemográficas de los extensionistas. En general, los extensionistas prefieren y utilizan más métodos grupales, seguidos de individuales. Los métodos de articulación institucional y de comunicación de masas quedaron ubicados en tercer y cuarto lugar respectivamente, tanto a nivel de preferencias como de uso. Se observó un desajuste importante entre los métodos preferidos y los más utilizados. Se identificaron tres tipos de extensionistas de acuerdo a los métodos que prefieren y utilizan, uno centrado en el trabajo individual y grupal con productores y otros dos que priorizan o relegan métodos individuales o grupales (según el caso), complementándolos con acciones de articulación institucional.

Palabras clave: extensión rural, métodos, herramientas, tipología.

\begin{abstract}
In contrast to the wide corpus of literature aimed at discussing different extension approaches or philosophies, there is scarce interest in the analysis of methods of rural extension. This quantitative study analyzes the types of rural extension methods that rural extension agents from nine countries prefer and use, and explores the relationship between the types of methods and extension agents' sociodemographic characteristics. Group methods are the most preferred and used, followed by individual ones. Interinstitutional coordination and mass media methods occupy third and fourth place respectively, in terms of preference and use. An important divergence between preferred and most used methods was identified. Results show the existence of three types of extension agents in terms of their extension methods, one focusing on individual and group work with farmers, and the other two which prioritize or relegate individual or group methods (depending on the case) and complement them with inter-institutional coordination ones.
\end{abstract} Keywords: rural extension, methods, tools, typology.

\section{Introducción}

En la actualidad existe una diversidad de formas posibles de pensar a la extensión rural, así como un amplio corpus de literatura académica e institucional orientado a discutir diferentes enfoques o filosofías de extensión (Landini, 2016; Leeuwis, 2004; Machado et al., 2006). En particular, en las últimas décadas, los autores tienden a destacar dos transformaciones 
centrales. Por un lado, el pasaje de enfoques orientados a la transferencia o difusión vertical de tecnologías, a propuestas centradas en la facilitación horizontal y participativa de aprendizajes (Cristóvão et al., 2012). Y por el otro, de una comprensión simplista y lineal de los sistemas productivos y de los procesos de innovación, a una mirada sistémica que reconoce el valor de múltiples actores y de diferentes formas de conocimiento (Hellin, 2012; Klerkx et al., 2012). En este trabajo, se utiliza la noción de 'extensión rural' como un concepto genérico referido a un conjunto de prácticas orientadas a la transferencia de tecnologías, la provisión de información, la educación y el fortalecimiento de los productores agropecuarios y de sus organizaciones, así como al impulso de procesos de desarrollo e innovación rural. En esta línea, se considera al asesoramiento técnico ('agricultural advisory services' en la literatura en inglés) como una de sus múltiples manifestaciones (Christoplos, 2010; Davis \& Sulaiman, 2016a).

En contraste con el amplio interés generado por la discusión relativa a los enfoques de extensión rural, la cuestión de los métodos de extensión ha despertado menos atención de los académicos. En general, cuando los autores aluden a los enfoques de extensión, refieren a los marcos filosóficos o a los encuadres de referencia que dan forma a los programas de acción; en tanto que cuando hablan de las metodologías o los métodos de extensión refieren a las técnicas, herramientas o mecanismos utilizados para alcanzar los fines propuestos (Davis \& Sulaiman, 2016b; Famuyiwa et al., 2017).

En la literatura académica existen diferentes formas de referirse a los métodos de extensión rural. Por un lado, encontramos múltiples referencias al uso de métodos difusionistas (Da Ros, 2012; Landini, 2012) y al uso de métodos participativos (Morais \& Callou, 2017; Rodríguez Espinosa \& Ramírez Gómez, 2015) como forma genérica de señalar la implementación de métodos consistentes con ambos enfoques de extensión. En paralelo, también existen trabajos que mencionan, describen o enumeran diferentes métodos o técnicas de trabajo específicas utilizadas frecuentemente por los extensionistas. Por ejemplo, Eberle \& Shroyer (2000) señalan que ya en el año 1930 los extensionistas rurales en Estados Unidos llevaban adelante demostraciones de métodos, exposiciones, visitas a predios de los productores, reuniones y producción de material impreso de divulgación de tecnologías agrícolas. Por su parte, Famuyiwa et al. (2017) señalan que los encuentros personales entre extensionistas y productores pueden tomar la forma de visitas a los predios, pero también pueden darse por teléfono o por visitas de los productores a las oficinas de extensión. A la vez, hacen referencia al uso de métodos grupales, dentro de las cuales mencionan las parcelas demostrativas, los días de campo donde se demuestran técnicas agrícolas y resultados a mayor escala pasando por diferentes estaciones, y las visitas a unidades productivas ubicadas en otras áreas junto con productores para conocer diferentes tecnologías y prácticas productivas.

En el contexto de esta diversidad de técnicas y estrategias para llevar adelante el trabajo de extensión rural, diferentes autores han llamado la atención sobre los beneficios y las limitaciones de cada una de ellas (Famuyiwa et al., 2017; Kingiri \& Nderitu, 2014). Así, existe consenso que no puede hablarse de un método que sea el mejor, sino que se necesita seleccionar de las diferentes alternativas las que sean más acordes con los objetivos, los costos y las características de los productores y del personal de extensión (David \& Asamoah, 2011; Davis \& Sulaiman, 2016b; Eberle \& Shroyer, 2000). En la misma línea, Kingiri \& Nderitu, (2014) argumentan que no existen ningún método de extensión que pueda ser considerado suficiente por sí mismo, por lo que es necesario complementar métodos para obtener mejores resultados.

En la bibliografía académica también existen trabajos que abordan tres propuestas específicas de cierto nivel de complejidad que han alcanzado reconocimiento. El método de 'Capacitación y Visitas' ('Training \& Visits' o 'T\&V' como es frecuentemente conocida en inglés), fue impulsado 
fuertemente por el Banco Mundial en las décadas del 80 y 90 del siglo pasado. La propuesta, enmarcada dentro de un enfoque vertical de difusión tecnológica (Cristóvão et al., 2012), se caracteriza por la capacitación periódica de agentes de extensión locales por parte de investigadores o expertos, para que visiten a productores de su zona de manera planificada cada 14 días a fin de difundir conocimientos y persuadir respecto de la adopción de las nuevas tecnologías (Musa et al., 2013).

Otro de los métodos más discutidos en la bibliografía son las 'Escuelas de Campo para Agricultores' (según la traducción de la Organización de las Naciones Unidas para la Agricultura y la Alimentación [FAO] del concepto 'Farmer Field Schools'). Esta es una propuesta formativa de carácter grupal para agricultores apoyada en principios del aprendizaje experiencial y la educación de adultos; en la cual, los propios participantes se convierten en investigadores de los problemas que los afectan para identificar o generar alternativas que sean apropiadas para sus circunstancias (Asiabaka, 2002). Dentro de este abordaje, los extensionistas (u otros agentes externos) ocupan el lugar de facilitadores y no de expertos (Davis \& Sulaiman, 2016a).

Finalmente, en la literatura también es posible encontrar múltiples referencias al enfoque 'Campesino a Campesino' (Holt Giménez, 2008). En este caso, la propuesta también parte de principios similares de los de las Escuelas de Campo, como los de aprendizaje experiencial y experimentación activa. No obstante, hay que reconocer que la metodología Campesino a Campesino no refiere a un modelo único sino a una diversidad de prácticas (Landini \& Vargas, 2020), que se caracterizan por apoyarse en la figura de promotores campesinos que comparten sus conocimientos y prácticas con otros agricultores a partir de técnicas basadas en la educación popular (Rosset et al., 2011). En este marco, los actores externos actúan con facilitadores del intercambio más que como expertos técnicos.

Ahora bien, en el marco de esta diversidad de métodos, algunos autores han presentado tipologías de métodos de extensión rural. La tipología más extendida hace la diferenciación de acuerdo a las audiencias, clasificando los métodos en individuales, grupales o de masas (Duveskog et al., 2011; Famuyiwa et al. 2017). Los métodos individuales refieren al vínculo uno a uno entre extensionistas y productores, incluyendo actividades como visitas a fincas, contactos telefónicos o encuentros en las oficinas de los profesionales. Los abordajes grupales, en tanto, hacen referencia al trabajo de extensionistas con grupos de productores, e incluyen actividades como reuniones grupales, capacitaciones, días de campo y parcelas demostrativas, así como propuestas más estructuradas como las Escuelas de Campo y la metodología Campesino a Campesino. Finalmente, los métodos de masas refieren a la comunicación de información a productores de manera genérica a través de medios de comunicación, como puede ser la radio, televisión o internet.

No obstante, cambios recientes en los enfoques de extensión rural invitan a incorporar un nuevo tipo de método, derivado de las propuestas que reconocen que los procesos de desarrollo e innovación rural poseen carácter sistémico y territorial, requiriendo de la articulación y coordinación entre múltiples actores. Así, surge un cuarto tipo de método, expresado como coordinación inter-actoral o interinstitucional, que puede cobrar forma a partir de la implementación de plataformas de innovación y de mesas de desarrollo territorial (Cristóvão et al., 2012; Davis \& Sulaiman, 2016b; Food and Agriculture Organization, 2015) o de ejercer roles como 'intermediario de innovación' (innovation broker), facilitando el vínculo entre diferentes actores en los sistemas de innovación (Rendón-Medel et al., 2015).

En este contexto, se reconoce que el estudio científico de los métodos de extensión rural ha despertado un interés limitado, y que la mayor parte de los trabajos que abordan el tema lo hacen a partir del análisis de métodos o técnicas específicas, de lo que se sigue el interés de 
llevar adelante un estudio de los tipos de métodos de extensión rural desde una perspectiva más amplia y abarcadora. Ahora bien, antes de pasar a la descripción detallada de los objetivos de este trabajo, resulta pertinente presentar una serie de consideraciones.

En primer lugar, es conveniente señalar que existe una importante diferencia entre preferir un método o un tipo de método de extensión (en el sentido de querer ponerlo en práctica) y utilizarlo realmente. Diferentes trabajos han evidenciado que pueden existir diferencias y tensiones entre los enfoques productivos y de extensión rural que prefieren los extensionistas y aquellos que les indican poner en práctica las instituciones de las que forman parte (AbduRaheem, 2014; Mahon et al., 2010). En este trabajo, se hipotetiza la posible existencia de tensiones entre los métodos que prefieren implementar los extensionistas, y aquellos que realmente usan como resultados de presiones de las instituciones y de los contextos en los que se encuentran inmersos. A nivel conceptual, esta consideración se deriva de reconocer que las condiciones estructurales constriñen los márgenes de acción de los sujetos, pero que a la vez estos poseen un margen de maniobra sostenido en su capacidad de agencia (Long, 2001), asociada en este caso a sus propias creencias y preferencias.

Por otra parte, también se reconoce que diferentes variables sociodemográficas pueden incidir tanto en los métodos que los extensionistas prefieren como aquellos que utilizan con más frecuencia. Atendiendo a la existencia de diferentes marcos institucionales y estructuras de gobernanza de la extensión en diferentes países (Davis et al., 2020), es esperable que los métodos difieran según los países donde se desempeñan los extensionistas. Por su parte, Landini et al. (2013) han señalado que el género, la edad y la experiencia pueden incidir en los enfoques de trabajo que adoptan los extensionistas rurales, en tanto que Nettle et al. (2018) registraron cambios en las estrategias se asesoramiento técnico como resultado de la formación de postgrado. Así, los antecedentes mencionados sugieren la posibilidad de asociaciones entre los métodos de extensión utilizados y preferidos por los extensionistas, y variables como edad, experiencia, nivel educativo, género y país. Incluso, es posible pensar no solo en la existencia de relaciones entre variables una a una, sino también en configuraciones más complejas analizables en términos de tipologías, por ejemplo, extensionistas de cierto género y franja etaria podrían ser más propensos a preferir ciertos métodos, o extensionistas de ciertos países con formación de postgrado a utilizar más frecuentemente otras.

Visto lo anterior, el presente artículo se propone como objetivos abordar, a partir de muestras de diferentes países, los tipos de métodos de extensión rural (individuales, grupales, de articulación institucional y de masas) preferidos y más utilizados por los extensionistas, indagar la relación existente entre diferentes variables sociodemográficas y los métodos de extensión preferidos y más utilizados, e identificar tipos de extensionistas rurales de acuerdo sus preferencia y uso de los diferentes tipos de métodos.

\section{Metodología}

Se realizó un estudio cuantitativo de tipo descriptivo y correlacional en nueve países (Argentina, Australia, Brasil, Chile, México, Nueva Zelanda, Nigeria, Paraguay y Sudáfrica), a partir de un convenio firmado entre la Universidad de la Cuenca del Plata y el Global Forum for Rural Advisory Services (GFRAS). La selección de los países siguió los criterios diversidad territorial y conveniencia. El primer criterio llevó a incluir países de África, América Latina y Oceanía. El segundo, consideró el acceso y los contactos locales dispuestos a colaborar con el estudio. Se diseñó y aplicó un cuestionario que abarcó diferentes dimensiones de las prácticas y los enfoques que utilizan los extensionistas. El presente artículo presenta de manera comparativa 
los resultados relativos a los métodos de extensión rural. Se informa que los datos referidos a Argentina fueron incluidos parcialmente en un artículo previo (Landini \& Beramendi, 2020) (sin que fueran comparados con otros países), y que los participantes del estudio recibieron una devolución descriptiva de los resultados de los cuestionarios (que no ha sido publicada ni presentada en eventos científicos, pero que potencialmente podría haber sido compartida en repositorios institucionales).

Los cuestionarios fueron enviados vía email por medio de la plataforma para encuestas SurveyMonkey ${ }^{\circledR}$, con excepción de algunos casos de Sudáfrica, donde también se recibieron respuestas en documentos escaneados. En Argentina, Chile y Paraguay la investigación recibió el apoyo de instituciones de extensión rural públicas de nivel nacional. En Brasil se obtuvo el apoyo de tres instituciones estaduales públicas de extensión y de extensionistas de una universidad. En el caso de México, se recurrió a integrantes del listado público del Sistema de Extensionismo Rural Mexicano (SER Mexicano). Finalmente, en Australia, Nigeria, Nueva Zelanda y Sudáfrica los cuestionarios se obtuvieron a través de diferentes organizaciones profesionales de extensionistas, de alcance nacional e internacional. Las respuestas fueron obtenidas entre 2017 y 2019, dependiendo el país.

El número de participantes que contestaron el cuestionario y sus características sociodemográficas se muestran en la Tabla 1. Como se observa, los tamaños de muestra son desiguales, lo que se debe tanto a la existencia de diferencias en la cantidad de extensionistas vinculados a las instituciones colaboradoras, como al diferente grado y tipo de apoyo recibido de cada una de ellas.

Tabla 1. Características sociodemográficas de los participantes

\begin{tabular}{lccccc}
\multicolumn{1}{c}{ País } & N & Género (\%) & $\begin{array}{c}\text { Edad } \\
\text { media }\end{array}$ & $\begin{array}{c}\text { Experiencia } \\
\text { (en años) }\end{array}$ & $\begin{array}{c}\text { Graduados } \\
\text { universitarios } \\
\text { (\%) }\end{array}$ \\
\hline Argentina & 591 & $\mathrm{H}: 65, \mathrm{M}: 35$ & 43,6 & 12,1 & 82,8 \\
Australia & 35 & $\mathrm{H}: 49, \mathrm{M}: 51$ & 48,5 & 17,1 & 97,1 \\
Brasil & 265 & $\mathrm{H}: 78, \mathrm{M}: 22$ & 47,1 & 18,3 & 79,7 \\
Chile & 187 & $\mathrm{H}: 56, \mathrm{M}: 43, \mathrm{O}: 1$ & 42,2 & 11,0 & 69,9 \\
México & 1509 & $\mathrm{H}: 77, \mathrm{M}: 23$ & 41,5 & 8,7 & 95,9 \\
Nigeria & 93 & $\mathrm{H}: 60, \mathrm{M}: 39, \mathrm{O}: 1$ & 43,9 & 12,0 & 100 \\
Nueva Zelanda & 17 & $\mathrm{H}: 82, \mathrm{M}: 18$ & 50,5 & 22,2 & 100 \\
Paraguay & 22 & $\mathrm{H}: 86, \mathrm{M}: 14$ & 41,0 & 14,1 & 81,0 \\
Sudáfrica & 42 & $\mathrm{H}: 69, \mathrm{M}: 31$ & 44,6 & 15,2 & 97,6 \\
Total/media & 2761 & $\mathrm{H}: 69, \mathrm{M}: 30, \mathrm{O}: 0$ & 44,8 & 14,5 & 89,3 \\
\hline
\end{tabular}

Notas: $\mathrm{n}=$ tamaño de la muestra; $\mathrm{H}=$ Hombre; $\mathrm{M}=$ Mujer; $\mathrm{O}=$ Otro

Para el presente estudio se incluyeron a los participantes que respondieron al menos una de las preguntas relativas a los tipos de métodos de extensión rural. Se excluyeron a quienes informaron tener cero años de experiencia como extensionistas, ya que carece de sentido que informaran sobre los tipos de métodos de extensión utilizados. Para fines de análisis estadístico los casos que indicaron tener más de cero y menos de un año de experiencia, se consideró como 0,5 años, y 51 para el único caso que informó tener "más de 50".

El cuestionario incluyó preguntas sociodemográficas y consignas relativas a diferentes aspectos de la práctica de la extensión. Dos fueron las consignas referidas a los tipos métodos de extensión, una relativa a las preferidas, y otra a las más utilizadas. En el cuestionario se presentaron a los participantes cuatro alternativas: 
1. Trabajo con productores individuales (o productores individuales y su familia)

2. Trabajo con grupos u organizaciones de productores

3. Trabajo de articulación o coordinación inter-institucional o inter-actoral

4. Trabajo con medios masivos de comunicación (difusión de técnicas productivas o información a través de medios como radio, teléfono celular, internet, redes sociales, televisión u otro similar). No se considera esta opción cuando el uso de teléfono, radio o internet es para comunicarse con productores individuales, grupos u organizaciones con los que se trabaja de manera personal

Respecto a la pregunta por los tipos de métodos preferidos, se pidió a los participantes que ordenaran las opciones de aquella que preferían más a la que preferían menos, asignándole 1 a la primera y 4 a la última, sin posibilidad de repetir u omitir el uso de ningún número. Para el caso de los tipos de métodos más utilizados, se repitió el procedimiento, pero permitiendo indicar las que no utilizaban con el número 0. Estas respuestas fueron convertidas en diferentes variables. Primero, se generaron dos variables nominales, una donde se identificaba el tipo de método preferido, y la otra el tipo de método más utilizado. En ambos casos se asignó valor a la variable cuando la respuesta no dejaba lugar a dudas respecto de la primera opción seleccionada.

Al mismo tiempo, por cada una de las dos preguntas se generaron cuatro variables ordinales, una por cada tipo de método, con los valores correspondieron al orden asignado por los participantes. Los cuatro valores de variable referidos a la preferencia fueron de 1 (mayor preferencia) a 4 (menos preferencia). En cambio, la variable relativa al uso fue de 1 (mayor uso) a 5 (ningún uso). Se consideraron valores perdidos a los casos donde los participantes no respetaron la consigna de seguir un orden y no repetir números.

Los datos fueron analizados con el software SPSS versión 25. La existencia de diferente número de participantes por país fue tomada en cuenta especialmente para los análisis estadísticos. Para la presentación y procesamiento de los datos se generaron diferentes tablas de resumen y se analizaron relaciones entre variables acorde a sus escalas de medida. Por último, se realizó un análisis de conglomerados a fin de construir una tipología de extensionistas rurales de acuerdo a sus preferencias y al uso de diferentes tipos de métodos de extensión. Para esto se utilizó el procedimiento de conglomerado bietápico del SPSS, ya que resulta apropiado para trabajar con variables de cualquier escala de medida (Pinazo \& Bautista-Vallejo, 2017) y de manera conjunta (cualitativas y cuantitativas) (Murillo, 2008), además de definir de manera automática el número óptimo de conglomerados (Vilà-Baños et al., 2014; Rubio-Hurtado \& Vilà-Baños, 2017).

Teniendo en cuenta el desbalance y en algunos casos el reducido número de participantes por país, se optó por realizar la tipología con los cuatro países con mayor número de casos, todos ellos latinoamericanos. Para este análisis se removieron los casos que no respondieron a las dos preguntas relativas a los métodos de trabajo de extensión. Debido a que el país con menor número de casos de los cuatro seleccionados fue Chile, con n=173, y para evitar sobrevaloración de tipos de extensionistas característicos de los países con mayor muestra (particularmente el caso de México), se decidió seleccionar al azar la misma cantidad de casos para Argentina, Brasil y México usando las funcionalidades del SPSS. Así, el número total de casos para el análisis fue de 692, incluyendo 173 casos por país. Después del procedimiento anterior y para evitar la posible influencia del orden de los casos en la base de datos, antes de hacer los análisis estadísticos correspondientes se reordenaron los casos al azar. 


\section{Resultados}

\section{Tipos de métodos de extensión con mayor nivel de preferencia}

En la Tabla 2 y en la Figura 1 se presenta el tipo de método de extensión seleccionado como primera preferencia por los encuestados según país $(n=2761)$. En ambas puede verse en general una mayor preferencia por métodos de tipo grupal con casi el 50\%, seguidos por métodos individuales (poco más de un tercio de los casos). En contraste, se observan preferencias mucho menores en el caso de métodos institucionales y especialmente de aquellos basados en medios masivos de comunicación.

Tabla 2. Tipos de métodos de extensión preferidos según muestras por país

\begin{tabular}{lcccc}
\multicolumn{1}{c}{ Países } & Individual (\%) & Grupal (\%) & $\begin{array}{c}\text { Institucional } \\
(\mathbf{\%})\end{array}$ & De masas (\%) \\
Argentina & 21,5 & 57,4 & 18,6 & 2,5 \\
Australia & 37,1 & 42,9 & 11,4 & 8,6 \\
Brasil & 40,4 & 42,6 & 14,3 & 2,6 \\
Chile & 65,8 & 23,5 & 10,2 & 0,5 \\
México & 29,8 & 55,9 & 10,3 & 4,0 \\
Nigeria & 22,6 & 41,9 & 15,1 & 20,4 \\
Nueva Zelanda & 47,1 & 47,1 & 0,0 & 5,9 \\
Paraguay & 9,1 & 77,3 & 13,6 & 0,0 \\
Sudáfrica & 38,1 & 35,7 & 21,4 & 4,8 \\
Medias de los países & 34,6 & 47,1 & 12,8 & 5,5 \\
\hline
\end{tabular}

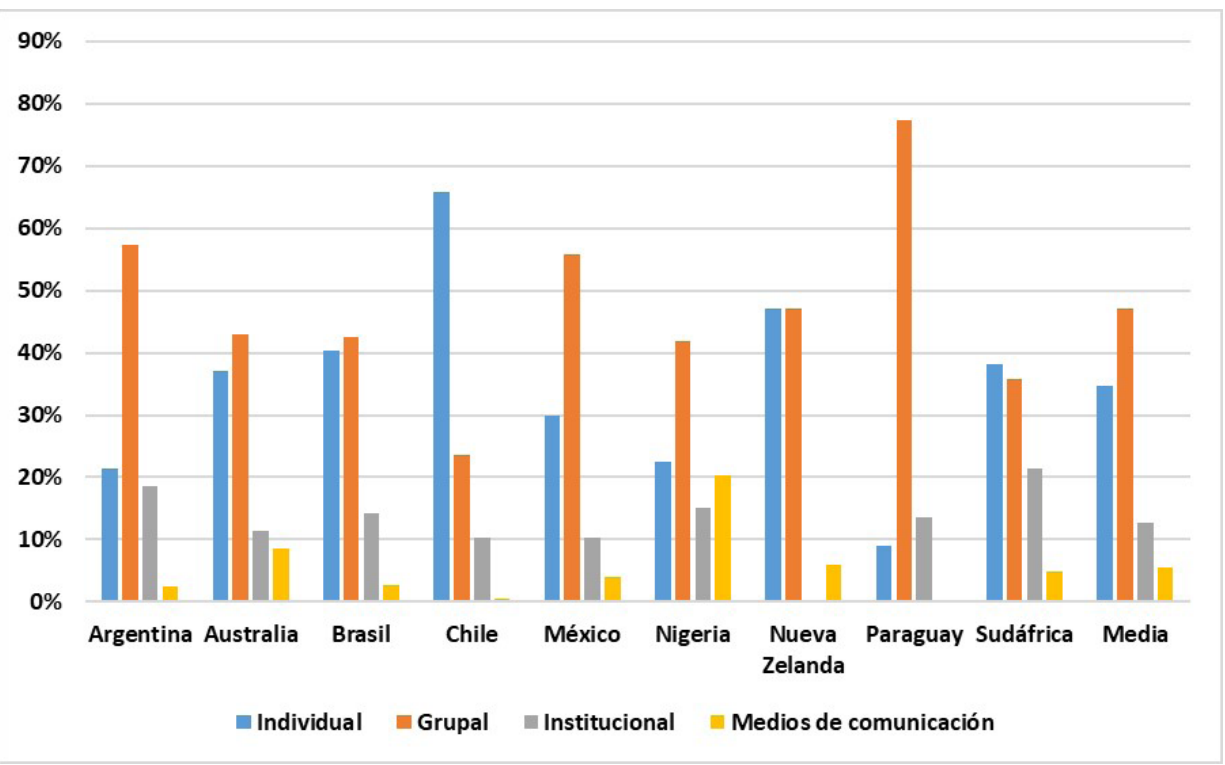

Figura 1. Tipos de métodos de extensión preferidos según muestras por país

En cuanto a las diferencias entre países, se observan cuatro países (Paraguay, Argentina, México y Nigeria) donde los extensionistas prefieren métodos de tipo grupal por sobre individuales o de otro tipo, destacándose claramente Paraguay. En contraste, en otros países, 
como Australia, Brasil, Nueva Zelanda y Sudáfrica parece existir un equilibrio entre ambas alternativas. Por su parte, el único caso en el que los extensionistas prefieren métodos de tipo individual por sobre el resto es Chile. Finalmente, Nigeria se destaca por tener un porcentaje mucho más alto que el resto de preferencia por el uso de medios masivos de comunicación como herramienta metodológica, aun sin ser su alternativa más seleccionada. Se destaca que las diferencias existentes entre los países son estadísticamente significativas $\left(\chi_{(24)}^{2}=260 ; p<.001\right)$.

\section{Tipos de métodos de extensión más utilizados}

En Tabla 3 y en la Figura 2 se presentan los métodos de extensión más utilizados por los encuestados según país, considerando los casos que tenían completa la información. Se observa que, en promedio, los métodos grupales son los más utilizados (poco más del 50\%), seguidos por los individuales (poco más de un tercio), en tanto que los métodos de articulación institucional y los de trabajo con medios masivos de comunicación fueron seleccionados con menor frecuencia.

Tabla 3. Tipos de métodos de extensión más utilizados por país ( $\mathrm{n}=2504)$

\begin{tabular}{lcccc}
\multicolumn{1}{c}{ Países } & Individual (\%) & Grupal (\%) & Institucional (\%) & De masas (\%) \\
Argentina & 24,4 & 58,8 & 14,2 & 2,6 \\
Australia & 25,7 & 45,7 & 17,1 & 11,4 \\
Brasil & 56,6 & 32,3 & 9,8 & 1,3 \\
Chile & 72,9 & 20,3 & 5,6 & 1,1 \\
México & 34,8 & 56 & 7,2 & 2,0 \\
Nigeria & 28,2 & 61,5 & 7,7 & 2,6 \\
Nueva Zelanda & 23,5 & 58,8 & 11,8 & 5,9 \\
Paraguay & 5,9 & 88,2 & 0,0 & 5,9 \\
Sudáfrica & 33,3 & 38,5 & 20,5 & 7,7 \\
Media de los países & 33,9 & 51,1 & 10,4 & 4,5 \\
\hline
\end{tabular}

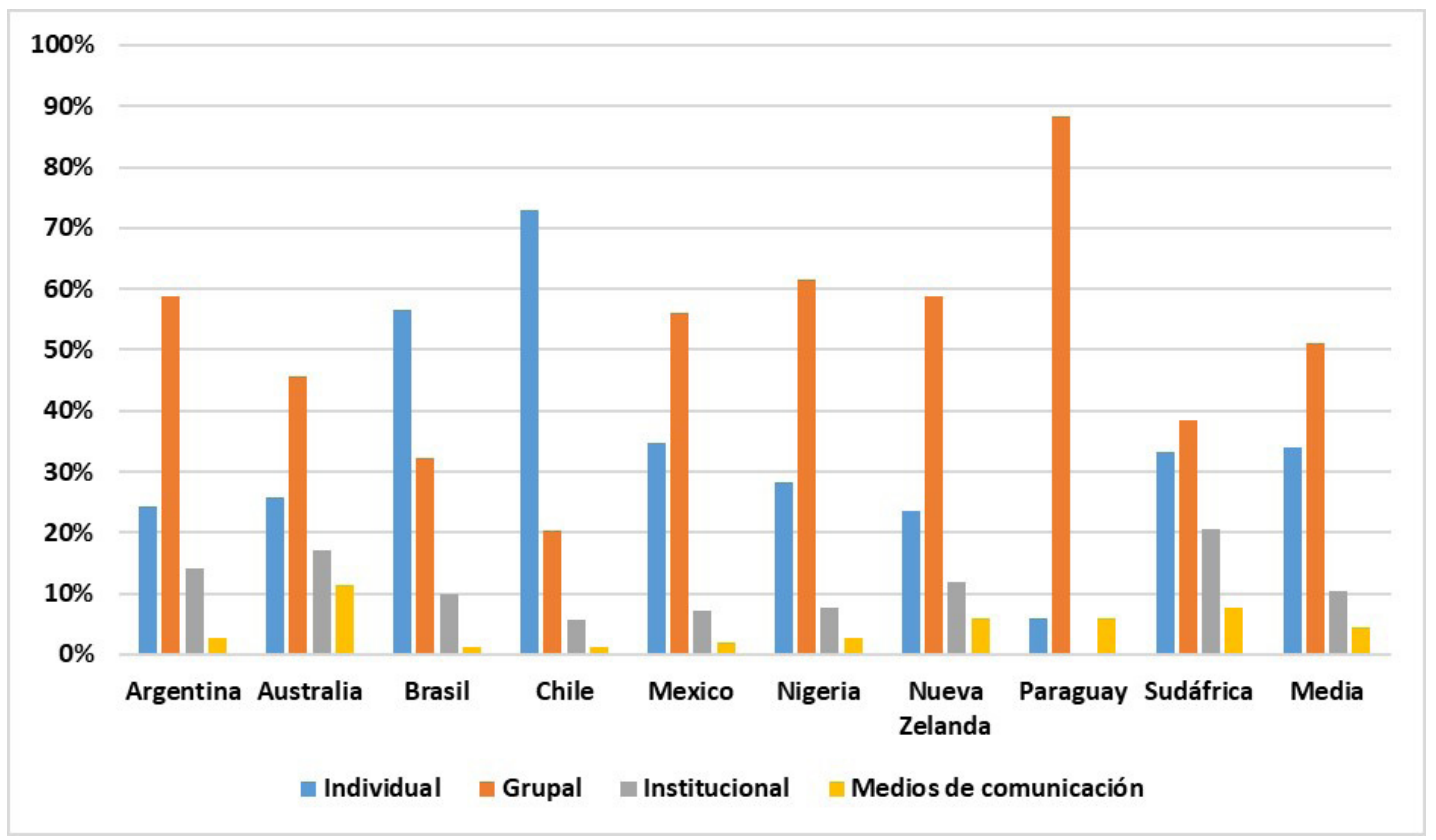

Figura 2. Tipos de métodos de extensión más utilizados por país 
Como puede observarse, los porcentajes referidos a los tipos de métodos más utilizados son similares, en términos generales, a aquellos obtenidos al preguntar por las preferencias. No obstante, a nivel de países específicos pueden verse algunas diferencias entre los tipos de métodos preferidos y los más utilizados. En Australia y Nueva Zelanda se observa que los métodos individuales son menos utilizados que lo que se desearía, lo que en el segundo país se complementa con un uso mucho más marcado de metodologías grupales. Por su parte, en Brasil se observa el fenómeno contrario, es decir, los métodos individuales son mucho más utilizados que los grupales, a la inversa de las preferencias de los extensionistas. Finalmente, llama la atención del caso de Nigeria, donde mientras más del 20\% de los encuestados prefiere utilizar medios masivos de comunicación como primer método de extensión, sólo el 2,6\% dice hacerlo. Se destaca que las diferencias existentes entre los países son estadísticamente significativas $\left(\chi_{(24)}^{2}=244 ; p<.001\right)$.

\section{Desajustes entre tipos de métodos de extensión preferidos y las más utilizados}

La existencia de porcentajes similares en las preferencias y en el uso de determinados métodos de extensión en un mismo país no implica que las personas utilicen aquellos que prefieren. Esto se debe a que los extensionistas pueden preferir un tipo de método, pero tener que usar otro, en tanto que esto podría compensarse con casos donde la preferencia y el uso sean inversos, generándose una interpretación de ajuste entre uso y preferencia, aun cuando esto no sea así a nivel individual. Para analizar esto, en la Tabla 4 se presentan por país los casos en los cuales existen diferencias entre el tipo de método preferido y el más utilizado.

Tabla 4. Diferencias entre tipos de métodos preferidos y más utilizados por país $(n=2504)$

\begin{tabular}{lccc}
\multicolumn{1}{c}{ Países } & Casos $(\mathbf{n})$ & $\begin{array}{c}\text { Casos } \\
\text { desajustados }(\mathbf{n})\end{array}$ & $\begin{array}{c}\text { Desajuste (\%) } \\
\text { Argentina }\end{array}$ \\
Australia & 537 & 117 & 21,8 \\
Brasil & 35 & 11 & 31,4 \\
Chile & 235 & 64 & 27,2 \\
México & 177 & 29 & 16,4 \\
Nigeria & 1369 & 311 & 22,7 \\
Nueva Zelanda & 78 & 33 & 42,3 \\
Paraguay & 17 & 7 & 41,2 \\
Sudáfrica & 17 & 2 & 11,8 \\
Totales y media de países & 39 & 16 & 41 \\
\hline
\end{tabular}

Nota: la media de porcentajes corresponde a la media de las medias de los diferentes países

Los resultados muestran que en más de un cuarto de los casos los tipos de métodos de extensión que utilizan los extensionistas con mayor frecuencia son diferentes a los que preferirían utilizar, existiendo diferencias estadísticamente significativas entre países $\left(\chi_{(8)}^{2}=35,6\right.$; $p<.001)$. En particular, se observa que en países como Nigeria, Nueva Zelanda y Sudáfrica el desajuste es superior al $40 \%$, en tanto que en Australia y Brasil los porcentajes están en torno al 30\%. En contraste, los países con menor desajuste son México, Argentina, Chile y Paraguay, destacándose este último. 


\section{Variables sociodemográficas y tipos de métodos de extensión}

Se analizó la existencia de relaciones estadísticas entre los tipos de métodos preferidos y los más utilizados, y diferentes variables sociodemográficas. Para evitar sesgar el resultado a partir de la amplia heterogeneidad del número de casos por país, se optó por realizar los análisis comparativos dentro de cada uno de estos. Las variables socioeconómicas incluidas fueron 'género', 'edad', 'experiencia como extensionistas' y 'nivel educativo'.

Los resultados muestran que los tipos de métodos preferidos y más utilizados varían según 'género', 'edad' y 'experiencia' en países puntuales. En Chile se observa que los hombres prefieren y usan más métodos individuales y menos grupales, en tanto que lo inverso sucede con las mujeres $\left(\chi_{(3)}^{2}=8,94, p=.030 ; \chi_{(3)}^{2}=8,15, p=.043\right)$. En Argentina los hombres utilizan más métodos individuales y grupales, en tanto que las mujeres hacen lo propio con los métodos de tipo institucional y de comunicación de masas $\left(\chi_{(3)}^{2}=8,36\right.$, $p=.039)$.

Para los casos de las variables 'edad' y 'experiencia', se en encontraron relaciones estadísticas con los métodos preferidos en México (respectivamente $F_{(3,1504)}=2352, p=.002$; $F_{(3,1504)}=725, p=.021$ ) y con los más utilizados en Argentina (respectivamente $F_{(3,533)}=1581$, $\left.p=.001 ; F_{(3,533)}=1689, p<.001\right)$. En México, los extensionistas de menor edad y experiencia son los que prefieren más métodos de tipo individual, mientras que los de mayor edad y experiencia prefieren más los métodos de comunicación de masas. En Argentina, los extensionistas más jóvenes y de menor experiencia utilizan con mayor frecuencia métodos individuales, en tanto que los de mayor edad y experiencia utilizan más métodos de tipo institucional.

Cabe resaltar que, al encontrarse relaciones estadísticas entre el género, la edad y la experiencia, con las variables referidas a los métodos de extensión únicamente en países puntuales, no es posible interpretarlas como una tendencia general, sino como relaciones específicas en dichos países. En cambio, el caso de la variable 'nivel educativo' es diferente, ya que se encuentra relacionada estadísticamente con los métodos de extensión preferidos y más utilizados en múltiples países (Tabla 5).

En términos generales, los resultados presentados en la Tabla 5 sugieren una tendencia general, en la cual los extensionistas que prefieren y usan métodos de articulación interinstitucional tienen mayor nivel educativo que quienes priorizan métodos de tipo individual o grupal. Esta tendencia se confirmó en varios de los países con mayor muestra (cuatro en el caso de las preferencias y tres en el de uso).

Esto podría interpretarse de dos maneras complementarias. En primer lugar, es posible que los extensionistas con mayor nivel educativo ocupen con mayor frecuencia puestos de cierto nivel jerárquico, lo que podría asociarse a mayores responsabilidades de trabajo de articulación interinstitucional. Así, no sería el nivel educativo en sí el que incrementaría la preferencia por y el uso de métodos de tipo institucional, sino el ocupar posiciones jerárquicas posibilitadas por la formación académica. No obstante, también sería posible pensar que un mayor nivel educativo implica un mayor conocimiento sobre teorías del desarrollo y de extensión rural que destacan el valor de la articulación interinstitucional y la coordinación de actores como elemento dinamizador de procesos de desarrollo e innovación. 
Tabla 5. Diferencias en las preferencias y uso de diferentes tipos de métodos de extensión según nivel educativo

\begin{tabular}{|c|c|c|c|c|}
\hline \multirow{2}{*}{ Países } & \multicolumn{2}{|c|}{ Preferencia } & \multicolumn{2}{|c|}{ Uso } \\
\hline & Kruskal Wallis & Rango Medio & Kruskal Wallis & Rango Medio \\
\hline $\begin{array}{c}\text { Argentina } \\
\mathrm{n}_{\text {Pref }}=587 \\
n_{\text {Uso }}=533\end{array}$ & $\chi_{(3)}^{2}=8,18^{*}$ & $\begin{array}{c}\text { alnd.: } 277 \\
\text { aGrupal: } 289 \\
\text { bInst.: } 327 \\
\text { abMedios: } 298\end{array}$ & $\chi_{(3)}^{2}=17,84^{* *}$ & $\begin{array}{c}\text { alnd.: } 240 \\
\text { aGrupal: } 265 \\
\text { bInst.: } 315 \\
\text { abMedios: } 302\end{array}$ \\
\hline $\begin{array}{c}\text { Chile } \\
n_{\text {Pref }}=183 \\
n_{\text {Uso }}=173\end{array}$ & $X_{(3)}^{2}=8,45^{*}$ & $\begin{array}{c}\text { alnd.: } 87,9 \\
\text { aGrupal: 90,9 } \\
\text { 'Inst.: 121 } \\
\text { abMedios: } 113\end{array}$ & $\chi_{(3)}^{2}=453$ & --- \\
\hline $\begin{array}{c}\text { México } \\
n_{\text {Pref }}=1490 \\
n_{\text {Uso }}=1353\end{array}$ & $\chi_{(3)}^{2}=21,2^{\star \star *}$ & $\begin{array}{c}\text { alnd.: } 727 \\
\text { aGrupal: } 735 \\
\text { 'Inst.: } 851 \\
\text { abMedios: } 758\end{array}$ & $\chi_{(3)}^{2}=21,42 * \star *$ & $\begin{array}{c}\text { alnd.: } 662 \\
\text { aGrupal: } 674 \\
\text { bInst.: } 795 \\
\text { aMedios: } 599\end{array}$ \\
\hline $\begin{array}{l}\text { Nigeria } \\
\mathrm{n}_{\text {Pref }}=91 \\
\mathrm{n}_{\text {Uso }}=76\end{array}$ & $\chi_{(3)}^{2}=1,77$ & --- & $\chi_{(3)}^{2}=1,12$ & --- \\
\hline $\begin{array}{c}\text { Paraguay } \\
\mathrm{n}_{\text {Pref }}=21 \\
\mathrm{n}_{\text {Uso }}=16\end{array}$ & $\chi_{(2)}^{2}=0,06$ & --- & $\chi_{(2)}^{2}=1,30$ & --- \\
\hline $\begin{array}{c}\text { Sudáfrica } \\
n_{\text {Pref }}=41 \\
n_{\text {Uso }}=38\end{array}$ & $\chi_{(3)}^{2}=10,39 *$ & $\begin{array}{c}\text { acInd.: } 22 \\
\text { abGrupal: } 17,1 \\
\text { dInst.: } 28,8 \\
\text { bMedios: 5,5 }\end{array}$ & $\chi_{(3)}^{2}=6,88$ & --- \\
\hline $\begin{array}{c}\text { Brasil } \\
\mathrm{n}_{\text {Pref }}=261 \\
\mathrm{n}_{\text {Uso }}=232\end{array}$ & $\chi_{(3)}^{2}=4,80$ & --- & $\chi_{(3)}^{2}=9,91 *$ & $\begin{array}{c}\text { alnd.: } 109 \\
\text { a Grupal: } 119 \\
\text { 'blnst.: } 149 \\
\text { ab Medios: } 142\end{array}$ \\
\hline $\begin{array}{c}\text { Australia } \\
\mathrm{n}_{\text {Pref }}=34 \\
\mathrm{n}_{\text {Uso }}=34\end{array}$ & $\chi_{(3)}^{2}=5,89$ & --- & $X_{(3)}^{2}=7,53$ & --- \\
\hline $\begin{array}{c}\text { Nueva Zelanda } \\
n_{\text {Pref }}=16 \\
n_{\text {Uso }}=16\end{array}$ & $\chi_{(3)}^{2}=0,90$ & --- & $\chi_{(3)}^{2}=1,81$ & --- \\
\hline
\end{tabular}

Notas: $\mathrm{n}_{\text {Pref }}=$ muestra 'preferencia'; $\mathrm{n}_{\text {uso }}=$ muestra 'uso'; * $=p<.05 ; * *=p<.01 ; * \star *=p<.001$. Las letras en superíndice indican grupos que difieren estadísticamente a un nivel de $p<.05$ (sin utilizar ajuste de Bonferroni).

\section{Tipología de extensionistas de países latinoamericanos seleccionados según preferencia y uso de métodos de extensión}

El procedimiento de análisis de conglomerados bietápico generó una solución de tres tipos. El Coeficiente de Silhouette de cohesión y separación se ubicó en 0,4 (indicando 0 que todos los casos se ubican equidistantes entre el centro de su conglomerado y el de otro conglomerado, y 1 que todos los casos están en el centro de su propio conglomerado). La observación de la figura correspondiente al criterio bayesiano de Schwarz (Rubio-Hurtado \& Vilà-Baños, 2017), confirmó que la solución de tres conglomerados resultaba la más razonable, combinando poder explicativo y simplicidad. Descriptivamente, el conglomerado 1 está compuesto por 
383 casos (55,3\%), el conglomerado 2 por 104 (15\%) y el conglomerado 3 por 205 (29.6\%); que al analizarlos con la técnica de Kruskal Wallis son diferentes estadísticamente a un nivel de $p<.001$ de acuerdo a las ocho variables introducidas en la clasificación.

Tabla 6. Tipos de métodos de extensión seleccionados como $1^{\mathrm{a}}$ y $2^{\mathrm{a}}$ opción por los diferentes tipos de extensionistas

\begin{tabular}{|c|c|c|c|c|c|c|c|c|c|c|}
\hline \multirow{2}{*}{$\begin{array}{c}\begin{array}{c}\text { Tipos de } \\
\text { métodos }\end{array} \\
\text { Preferencias }\end{array}$} & \multirow{2}{*}{ KW: } & \multicolumn{3}{|c|}{ Tipo 1} & \multicolumn{3}{|c|}{ Tipo 2} & \multicolumn{3}{|c|}{ Tipo 3} \\
\hline & & $\begin{array}{c}1^{\mathrm{a}} \\
(\%)\end{array}$ & $\begin{array}{c}2^{\mathrm{a}} \\
(\%)\end{array}$ & RM & $\begin{array}{c}1^{\mathrm{a}} \\
(\%)\end{array}$ & $\begin{array}{c}2^{\mathrm{a}} \\
(\%)\end{array}$ & RM & $\begin{array}{c}1^{\mathrm{a}} \\
(\%)\end{array}$ & $\begin{array}{c}2^{\mathrm{a}} \\
(\%)\end{array}$ & RM \\
\hline Individual & $\chi_{(2)}^{2}=405^{*}$ & 56,1 & 40,5 & $246^{a}$ & 56,7 & 26,0 & $276^{a}$ & 0,0 & 5,9 & $570^{b}$ \\
\hline Grupos & $\chi_{(2)}^{2}=251 *$ & 43,9 & 50,1 & $333^{a}$ & 3,8 & 15,4 & $594^{b}$ & 70,7 & 27,8 & $247^{c}$ \\
\hline Institucional & $\chi_{(2)}^{2}=326^{*}$ & 0,0 & 9,1 & $456^{a}$ & 28,8 & 33,7 & $281^{b}$ & 29,3 & 61,0 & $174^{c}$ \\
\hline Medios & $\chi_{(2)}^{2}=105^{*}$ & 0,0 & 0,3 & $394^{a}$ & 10,6 & 25,0 & $232^{b}$ & 0,0 & 5,4 & $316^{c}$ \\
\hline \multicolumn{11}{|l|}{ Uso } \\
\hline Individual & $\chi_{(2)}^{2}=348^{*}$ & 64,0 & 34,7 & $254^{a}$ & 63,5 & 22,1 & $285^{a}$ & 4,9 & 14,1 & $550^{b}$ \\
\hline Grupos & $\chi_{(2)}^{2}=259 *$ & 35,5 & 62,4 & $349^{a}$ & 8,7 & 14,4 & $577^{b}$ & 76,6 & 22,4 & $224^{c}$ \\
\hline Institucional & $\chi_{(2)}^{2}=286^{*}$ & 0,5 & 2,6 & $451^{a}$ & 17,3 & 51,0 & $257^{b}$ & 18,0 & 60,5 & $197^{c}$ \\
\hline Medios & $\chi_{(2)}^{2}=66^{*}$ & 0,0 & 0,0 & $391^{a}$ & 10,6 & 12,5 & $247^{b}$ & 0,0 & 2,4 & $313^{c}$ \\
\hline
\end{tabular}

Notas: KW = Kruskal Wallis; RM = Rango Medio (menor RM indica mayor preferencia o uso); * $p<.001$; las letras en superíndice, que deben leerse horizontalmente, indican grupos que difieren estadísticamente a un nivel de $p<.01$ (sin utilizar ajuste de Bonferroni).

Tabla 7. Variables sociodemográficas asociadas a los diferentes tipos de extensionistas

\begin{tabular}{|c|c|c|c|c|c|}
\hline & \multirow{2}{*}{ Estadístico } & & \multicolumn{3}{|c|}{ Tipos (\%) } \\
\hline & & & 1 & 2 & 3 \\
\hline \multirow[t]{4}{*}{ País } & \multirow{4}{*}{$\chi_{(6)}^{2}=81.4^{\star \star \star}$} & Argentina & 17,5 & 19,2 & 42,0 \\
\hline & & Brasil & 28,7 & 20,2 & 20,5 \\
\hline & & Chile & 28,5 & 44,2 & 8,8 \\
\hline & & México & 25,3 & 16,3 & 28,8 \\
\hline \multirow[t]{2}{*}{ Sexo } & \multirow{2}{*}{$X_{(2)}^{2}=7,21 *$} & Hombre & 71,7 & 58,3 & 66,3 \\
\hline & & Mujer & 28,3 & 41,7 & 33,7 \\
\hline Edad & $F_{(2,689)}=1,39$ & --- & --- & --- & --- \\
\hline Experiencia & $F_{(2,689)}=2,01$ & --- & --- & --- & --- \\
\hline Nivel educativo & $\mathrm{KW}: \chi^{2}=4,42$ & --- & --- & --- & --- \\
\hline
\end{tabular}

Notas: $*=p<.05 ; * *=p<.01 ; * * *=p<.001$. En el caso de la variable 'sexo' no se consideró la opción 'otro' dado que correspondía únicamente a dos casos.

De acuerdo a las Tablas 6 y 7, se observa que el tipo de extensionista 1 se caracteriza por preferir y utilizar métodos individuales y grupales (cuando un tipo es seleccionado como primera opción, el otro es seleccionado como segundo), relegando de sus preferencias y usos métodos de tipo institucional y de medios de comunicación masiva. En términos cuantitativos se trata del grupo más numeroso (55,3\% de los casos). A nivel sociodemográfico está compuesto por un mayor porcentaje de hombres en relación a los otros dos tipos de extensionistas.

El tipo de extensionista 2 es mucho más pequeño cuantitativamente (15\% de los casos), se caracteriza por extensionistas que predominantemente prefieren y usan métodos individuales, con un importante porcentaje de métodos de tipo institucional. En paralelo, llama fuertemente la atención la escasa preferencia y el escaso uso de métodos grupales (consideradas solo en el $19,2 \%$ de los casos como primera o segunda opción, cuando este porcentaje supera el $90 \%$ en 
los conglomerados restantes). Este grupo se encuentra conformado por un mayor porcentaje de mujeres que los dos restantes, y es más frecuente en Chile.

Por último, el tipo 3 se caracteriza por una clara preferencia y un uso frecuente de métodos grupales, lo que es complementado generalmente con métodos de tipo institucional. En contraste, los métodos individuales ocupan un rol periférico. Los integrantes de este conglomerado corresponden con mayor frecuencia a casos de Argentina, y con menor frecuencia a Chile.

En general se observa que el tipo 1 de extensionistas prioriza métodos grupales e individuales (en contacto directo con los productores en terreno) en tanto que relega la articulación institucional, mientras que los dos restantes complementan cada tipo de método de contacto directo (individual el tipo 2 y grupal el tipo 3) con la articulación institucional.

\section{Discusión}

En esta investigación se observó que los métodos de tipo grupal son los más utilizados y los que cosechan mayores preferencias entre los extensionistas de la mayor parte de los países estudiados. Este resultado es consistente con una investigación realizada en 10 países latinoamericanos, en el cual se encontró que el trabajo con grupos de productores y el fortalecimiento de las organizaciones está en el núcleo de los enfoques de extensión utilizados (Landini, 2016).

En general, se ha argumentado que los métodos grupales permiten reducir el costo de atención por productor (Davis \& Sulaiman, 2016a; Ndoro et al., 2014) y alcanzar a mayor cantidad de productores (Wellard et al., 2013). En este sentido, es frecuente que las agencias gubernamentales prioricen la atención de productores agrupados (Alimirzaei \& Asady, 2011). Incluso, resulta claro que los métodos grupales pueden mejorar el clima y los procesos de aprendizaje de los productores (Famuyiwa et al., 2017) ya que esto les permite compartir conocimientos y experiencias, como se observa en las 'Escuelas de Campo' y en la metodología 'Campesino a Campesino'. No obstante, como muestran Sanga et al. (2013) en el caso de Tanzania, también es posible que los servicios extensión prioricen formatos individuales aun en el contexto de recursos limitados. Así, es probable que la decisión de priorizar uno u otro no se deba solo a una evaluación de la relación costo/beneficio, sino también a tradiciones institucionales, a preferencias de autoridades de turno y a demandas de productores y otros actores.

Por su parte, el estudio realizado encontró que los métodos individuales se ubican en segundo lugar en el promedio de los países, tanto a nivel de preferencias como de uso. En general, los autores destacan que se trata de un tipo de método que requiere de mayor costo por productor, pero que aun así tiene gran importancia en la tarea de extensión (Davis \& Sulaiman, 2016a; Famuyiwa et al., 2017), ya que permite al extensionista ayudar a los productores a resolver problemas complejos referidos a situaciones específicas (Eberle \& Shroyer, 2000). En la misma línea, Landini (2012) señala que los pequeños productores paraguayos reclaman que los extensionistas visiten sus fincas para ofrecerles un asesoramiento técnico personalizado que se centre en sus problemas específicos. En particular, sería esperable un predominio de métodos individuales cuando los servicios de extensión se encuentren orientados a la demanda, y los productores demanden asesoramiento individual, algo potencialmente más probable en el marco de la extensión privada.

Por su parte, los métodos institucionales y de medios de comunicación de masas se ubicaron entre tercer y cuarto lugar tanto a nivel de preferencias como de uso en la media de los países participantes, con una gran distancia cuantitativa respecto de los métodos de tipo grupal e 
individual. En este sentido, se observa que buena parte de la tarea de extensión rural se realiza 'en terreno' en contacto directo con los productores.

Respecto de los métodos de tipo institucional, debe reconocerse que solo han adquirido interés más recientemente a partir de transformaciones acontecidas en las teorías del desarrollo y de la innovación, particularmente a partir de abordajes como el desarrollo territorial, las plataformas de innovación y la valorización de figuras como la del 'intermediario de innovación' (Cristóvão et al., 2012; Davis \& Sulaiman, 2016b; Rendón-Medel et al., 2015). Posiblemente, su menor porcentaje de preferencia y uso se explica tanto por su novedad como por tratarse de prácticas que, si bien son de gran importancia en términos de procesos de desarrollo e innovación, no requiere que sean llevadas adelante por un alto porcentaje de extensionistas. El hecho de que aquellos que priorizan métodos de articulación inter-institucional o inter-actoral tengan mayor nivel educativo que el resto puede sugerir que son las autoridades institucionales o personal técnico especializado los que se ocupan de estas labores.

En la literatura académica diversos autores han llamado la atención sobre el potencial de los medios de comunicación masivos como forma de difundir conocimientos e información agropecuaria, algo que se ha visto fortalecido en las últimas décadas con la aparición de internet y los teléfonos inteligentes (González Tena et al., 2015). En particular se ha destacado su bajo costo y su posibilidad de alcanzar a grandes cantidades de productores (Eberle \& Shroyer, 2000), así como su potencial para generar conciencia sobre diferentes situaciones y problemas (Famuyiwa et al., 2017). En este contexto, Ilama la atención lo poco que los métodos de comunicación de masas son utilizados por los extensionistas encuestados, lo que lleva a pensar en las oportunidades que se pierden de difundir conocimiento e información de manera amplia y a bajo costo. No obstante, también debe reconocerse que, dado su alcance, sería razonable que solo un porcentaje reducido de extensionistas priorizara métodos de comunicación de masas, ocupándose la mayoría de trabajar a nivel individual o grupal con productores.

Los resultados obtenidos también evidenciaron diferencias entre países. En particular, se observó que Chile es el único país donde los métodos individuales se ubican en el primer lugar de las preferencias, y que, tanto en Brasil como en Chile, se utilizan con mayor frecuencia que los métodos grupales. Esto resulta llamativo, ya que ambos casos los integrantes de la muestra son contratados por instituciones públicas para ofrecer servicios de extensión gratuitos, lo que haría suponer que se priorizarían métodos grupales para aumentar la cantidad de productores que reciben apoyo. Sin embargo, como se indicó, esto podría estar relacionado con tradiciones institucionales específicas o con preferencias de las autoridades respectivas.

Otro tema abordado fue el desajuste entre las preferencias y los métodos más utilizados por los extensionistas en los diferentes países. Los resultados mostraron que en tres de los países el desbalance supera el 40\%, y en dos adicionales se acerca al 30\%. La revisión bibliográfica no permitió identificar ninguna publicación académica que analice en profundidad la diferencia entre los métodos preferidos y los más utilizados en diferentes países, por lo que este resultado constituye un aporte singular de este artículo. En particular, la existencia de valores altos de desajuste entre métodos preferidos y más utilizados causa cierta preocupación, en tanto podría estar evidenciando que los extensionistas no están convencidos de los métodos que utilizan (lo harían por obligación o por condicionamientos externos). Además, es posible que los extensionistas se vean llevados a utilizar métodos para los cuales no se sienten suficientemente formados. Así, el desajuste entre métodos preferidos y más utilizados podría tener impacto en la calidad de la labor de los extensionistas, lo que hace importante que las instituciones evalúen estas diferencias y puedan asignar a los extensionistas tareas acordes con su perfil y preferencias.

Otro aporte de este artículo fue la construcción de una tipología de extensionistas rurales, según los métodos que prefieren y utilizan. En ningún otro estudio se identificó un trabajo 
similar. Los resultados muestran que el tipo más numeroso (compuesto por el $55 \%$ de los extensionistas) corresponde a los extensionistas 'de terreno', que alternan métodos individuales y grupales, por lo que su trabajo gira en torno al vínculo directo con los productores, sin incluir trabajos ni de articulación institucional ni de medios de comunicación entre las dos primeras elecciones de uso y preferencia. En paralelo, se identificó un grupo más pequeño (15\%), que prioriza métodos individuales y los complementa con institucionales y de medios masivos de comunicación, relegando de manera llamativa los métodos de tipo grupal. Finalmente, el tercer tipo, correspondiente al 30\% de la muestra, se caracteriza por trabajar fundamentalmente de manera grupal con los productores, complementándose esta actividad con métodos institucionales, generalmente seleccionados en segundo lugar. De acuerdo a múltiples autores, es fundamental tener la flexibilidad para seleccionar los métodos de extensión más apropiados para cada contexto, por lo que se reconoce la importancia de la flexibilidad tanto de los extensionistas como de las instituciones para seleccionar qué tipo de métodos utilizar (Eberle \& Shroyer, 2000; Kingiri \& Nderitu, 2014).

Antes de finalizar es importante señalar las limitaciones de la presente investigación. A nivel cuantitativo cabe destacar que el tamaño de las muestras no es igual en todos los países, por lo que es conveniente tomar con mayor cuidado los resultados de aquellos con muestras más pequeñas. Además, como las muestras fueron recolectadas con el apoyo de instituciones y redes profesionales específicas, no resulta apropiado considerarlas como representativas de todos los extensionistas de dichos países.

A la vez, al haberse utilizado como base un cuestionario con preguntas cerradas, no fue posible explorar respuestas a las siguientes interrogantes: ¿De qué manera los participantes definen y caracterizan los diferentes métodos? (podrían hacerlo diferente a lo descripto por la bibliografía) ¿Por qué los participantes prefieren y utilizan más unos tipos de métodos que otros?, ¿Qué razones tienen para preferir en un número considerable de casos un método y usar otro? Así, resultaría de interés complementar el presente estudio con un trabajo cualitativo que permita reconstruir las perspectivas y discursos de los extensionistas en relación a los métodos de extensión, buscando respuestas a las preguntas no abordadas en esta investigación.

Por su parte, es claro que el presente estudio puso el foco en los propios extensionistas, en sus preferencias y en los métodos que usan. Sin embargo, no se analizaron diferentes aspectos contextuales que pueden incidir fundamentalmente en los métodos de extensión utilizados, como los tipos de institucionales de pertenencia, el objetivo de su trabajo como extensionistas o asesores técnicos, y las demandas específicas de los productores. En este sentido, sería conveniente profundizar el análisis estudiando los vínculos y determinaciones mutuas que existen entre estos diferentes aspectos.

Finalmente, en este artículo se señaló la importancia de complementar métodos para alcanzar diferentes finalidades y de actuar de manera flexible para responder a diferentes contextos. Hasta cierto punto, la construcción de la tipología de extensionistas según métodos preferidos y utilizados permitió analizar aquellos que suelen utilizarse de manera articulada. No obstante, es claro que el presente trabajo no exploró con suficiente profundidad este tipo de dinámicas, por lo que se trata de un enfoque que resultaría valioso de considerar en trabajos futuros.

\section{Conclusiones}

En este artículo se estudiaron tipos de métodos que prefieren y utilizan extensionistas rurales de nueve países. Los resultados evidenciaron que, en el promedio de países, los métodos grupales son los que los extensionistas prefieren y más utilizan. Los métodos individuales se 
ubicaron en un segundo lugar, e incluso fueron los más utilizados en países como Chile y Brasil. Resulta claro que los métodos de tipo individual, si bien requieren más recursos por productor, también permiten un trabajo más personalizado y adaptado a las necesidades individuales. Los métodos de articulación institucional y de medios de comunicación de masas quedaron ubicados en tercer y cuarto lugar respectivamente, tanto a nivel de preferencias como de uso. En el caso de los métodos de comunicación de masas, llama la atención el escaso uso, teniendo en cuenta su potencial para alcanzar a grandes cantidades de productores a bajo costo.

El estudio también permitió reconocer un desajuste importante en algunos países entre los métodos que los extensionistas prefieren y los que terminan utilizando, situación que podría llevar a que el trabajo de los extensionistas no pueda expresar su máximo potencial.

Por último, se identificaron tres tipos de extensionistas de acuerdo a los métodos que prefieren y utilizan, uno centrado en el trabajo individual y grupal con productores a nivel de campo (el más numeroso), y otros dos que priorizan alternativamente métodos individuales o grupales, complementándolos fundamentalmente con métodos de articulación institucional.

\section{Agradecimientos}

El trabajo de investigación en que se basa el artículo fue financiado por la Universidad de la Cuenca del Plata en el marco del proyecto "Evaluación de las concepciones de extensión rural de extensionistas de diferentes países" (2016-Junio a 2019-Diciembre). Dicho proyecto fue implementado en el marco de un convenio firmado por la Universidad de la Cuenca del Plata y el Global Forum for Rural Advisory Services (GFRAS).

\section{Referencias}

Abdu-Raheem, K. (2014). Exploring the role of agricultural extension in promoting biodiversity conservation in KwaZulu-Natal province, South Africa. Agroecology and Sustainable Food Systems, 38(9), 1015-1032. http://dx.doi.org/10.1080/21683565.2014.899283

Alimirzaei, E., \& Asady, A. (2011). Individual factors affecting farmers' motivation to participate in date growers' organizations in Khuzestan. Research Journal of Applied Sciences, Engineering and Technology, 3(8), 725-730. Recuperado el 23 de mayo de 2020, de https://maxwellsci. com/print/rjaset/v3-725-730.pdf

Asiabaka, C. (2002). Promoting sustainable extension approaches: Farmer Field School (FFS) and its role in sustainable agricultural development in Africa. International Journal of Agriculture \& Rural Development, 3(1), 46-53. http://dx.doi.org/10.4314/ijard.v3i1.2518

Christoplos, I. (2010). Mobilizing the potential of rural and agricultural extension. Roma: FAO. Recuperado el 23 de mayo de 2020, de http://www.fao.org/3/i1444e/i1444e.pdf

Cristóvão, A., Koutsouris, A., \& Kügler, M. (2012). Extension systems and change facilitation for agricultural and rural development. In I. Darnhofer, D. Gibbon \& B. Dedieu (Eds.), Farming systems research into the 21st century: The new dynamic (pp. 201-227). Dordrecht, Países Bajos: Springer. http://dx.doi.org/10.1007/978-94-007-4503-2_10.

Da Ros, C. (2012). Gênese, desenvolvimento, crise e reformas nos serviços públicos de extensão rural durante a década de 1990. Mundo Agrario, 13(25). Recuperado el 23 de mayo de 2020, de https://www.mundoagrario.unlp.edu.ar/article/view/MAv13n25a04/html

David, S., \& Asamoah, C. (2011). Video as a tool for agricultural extension in Africa: a case study from Ghana. International Journal of Education and Development Using ICT, 7(1), 26-41. Recuperado el 23 de mayo de 2020, de https://www.learntechlib.org/p/42255/ 
Davis, K. y Sulaiman, R. (2016b). Module 2: Extension methods and tools. Lindaw, Suiza: GFRAS.

Davis, K., \& Sulaiman, R. (2016a). Overview of extension philosophies and methods. Note 0. Lausanne, Suiza: Global Forum for Rural Advisory Services (GFRAS).

Davis, K., Babu, S. C., \& Ragasa, C. (Eds.), (2020). Agricultural extension global status and performance in selected countries. Washington: International Food Policy Research Institute. Recuperado el 23 de mayo de 2020, de http://ebrary.ifpri.org/utils/getfile/collection/ p15738coll2/id/133965/filename/134181.pdf

Duveskog, D., Friis-Hansen, E., \& Taylor, E. W. (2011). Farmer field schools in rural Kenya: a transformative learning experience. The Journal of Development Studies, 47(10), 1529-1544. http://dx.doi.org/10.1080/00220388.2011.561328

Eberle, W., \& Shroyer, J. (2000). Are traditional extension methodologies extinct or just endangered? Journal of Natural Resources and Life Sciences Education, 29, 135-140. Recuperado el 23 de mayo de 2020, de https://www.agronomy.org/files/jnrlse/issues/2000/e00-01.pdf

Famuyiwa, B., Olaniyi, O., \& Adesoji, S. (2017). Appropriate extension methodologies for agricultural development in emerging economies. In W. Ganpat, R. Dyer \& W. Isaac (Eds.), Agricultural development and food security in developing nations (pp. 82-105). Hershey, Estados Unidos: IGI Global. http://dx.doi.org/10.4018/978-1-5225-0942-4.ch004.

Food and Agriculture Organization - FAO. (2015). Desarrollo territorial, innovación y comunicación rural hacia un enfoque integrado en apoyo a la agricultura familiar. Brasilia: FAO. Recuperado el 23 de mayo de 2020, de http://www.fao.org/3/a-i6225s.pdf

González Tena, P., Rendón Medel, R., Sangerman-Jarquín, D., Cruz Castillo, J., \& Díaz José, J. (2015). Extensionismo agrícola en el uso de tecnologías de la información y comunicación (TIC) en Chiapas y Oaxaca. Revista Mexicana de Ciencias Agrícolas, 6(1), 175-186. Recuperado el 23 de mayo de 2020, de http://www.scielo.org.mx/pdf/remexca/v6n1/v6n1a15.pdf

Hellin, J. (2012). Agricultural extension, collective action and innovation systems: Lessons on network brokering from Peru and Mexico. Journal of Agricultural Education and Extension, 18(2), 141-159. http://dx.doi.org/10.1080/1389224X.2012.655967

Holt Giménez, E. (2008). Campesino a campesino. Voces de Latinoamérica - Movimiento Campesino para la Agricultura Sustentable. Managua: SIMAS. Recuperado el 23 de mayo de 2020, de http://www.simas.org.ni/media/1335459671_CaC_Voces_latinoamerica.pdf

Kingiri, A., \& Nderitu, S. (2014). Assessment of extension and advisory methods and approaches to reach rural women (examples from Kenya). Nairobi, Kenya: Modernizing Extension and Advisory Services (MEAS). Recuperado el 23 de mayo de 2020, de https://meas.illinois.edu/ wp-content/uploads/2017/02/MEAS-EVAL-2014-EAS-Reaching-Rural-Women-Report-KenyaKingiri-Nderitu-July-2014.pdf

Klerkx, L., Van Mierlo, B., \& Leeuwis, C. (2012). Evolution of systems approaches to agricultural innovation: concepts, analysis and interventions. In I. Darnhofer, D. Gibbon \& B. Dedieu (Eds.), Farming systems research into the 21st century: the new dynamic (pp. 457-483). Dordrecht, Países Bajos: Springer. http://dx.doi.org/10.1007/978-94-007-4503-2_20

Landini, F. (2012). Problemas en la extensión rural paraguaya: modelos de extensión en la encrucijada. Cuadernos de Desarrollo Rural, 9(69), 127-149. Recuperado el 23 de mayo de 2020, de http://www.scielo.org.co/pdf/cudr/v9n69/v9n69a07.pdf

Landini, F. (2016). Concepción de extensión rural en 10 países latinoamericanos. Andamios, 13(30), 211-236. Recuperado el 23 de mayo de 2020, de http://www.scielo.org.mx/pdf/ anda/v13n30/1870-0063-anda-13-30-00211.pdf 
Landini, F., \& Beramendi, M. (2020). Objetivos priorizados y metodologías más utilizadas por los extensionistas rurales argentinos. Cuadernos de Desarrol/o Rural, 17(85), http://dx.doi. org/10.11144/Javeriana.cdr17.opmu

Landini, F., \& Vargas, G. L. (2020). Evaluación de los problemas que limitan el impacto de la extensión pública en el oriente de Guatemala. Revista de Economia e Sociologia Rural, 58(1), e192529. http://dx.doi.org/10.1590/1806-9479.2020.192529

Landini, F., Bianqui, V., \& Crespi, M. (2013). Evaluación de las creencias sobre extensión rural de los extensionistas paraguayos. Psiencia, 5(1), 3-14. http://dx.doi.org/10.5872/psiencia. v5i1.100

Leeuwis, C. 2004. Communication for rural innovation. Rethinking agricultural extension. Oxford: Blackwell Science.

Long, N. (2001). Development sociology: actor perspectives. Londres: Routledge.

Machado, J., De Hegedüs, P., \& Silveira, L. (2006). Estilos de relacionamento entre extensionistas e produtores: desde uma concepção bancária até o" empowerment. Ciência Rural, 36(2), 641-647. http://dx.doi.org/10.1590/S0103-84782006000200044

Mahon, M., Farrell, M., \& McDonagh, J. (2010). Power, positionality and the view from within: Agricultural advisers' role in implementing participatory extension programmes in the Republic of Ireland. Sociologia Ruralis, 50(2), 104-120. http://dx.doi.org/10.1111/j.14679523.2010.00505.x

Morais, J., \& Callou, A. (2017). Metodologias participativas e desenvolvimento local: a experiência do Projeto Dom Hélder Câmara no assentamento Moacir Lucena. Interações (Campo Grande), 18(1), 165-177. http://dx.doi.org/10.20435/1984-042x-2017-v.18-n.1(13)

Murillo, F. (2008). Los modelos multinivel como herramienta para la investigación educativa. Magis, Revista Internacional de Investigación en Educación, 1(1), 45-62. Recuperado el 23 de mayo de 2020, de https://www.redalyc.org/pdf/2810/281021687004.pdf

Musa, Y., Aboki, E., \& Audu, I. (2013). The limitations and implications of training and visit (T\&V) extension system in Nigeria. Journal of Agriculture and Sustainability, 4(1), 67-76. Recuperado el 23 de mayo de 2020, de https://www.infinitypress.info/index.php/jas/article/ viewFile/214/182

Ndoro, J., Mudhara, M., \& Chimonyo, M. (2014). Livestock extension programmes participation and impact on smallholder cattle productivity in Kwazulu-Natal: A propensity score matching approach. South African Journal of Agricultural Extension, 42(2), 62-80. Recuperado el 23 de mayo de 2020, de http://www.scielo.org.za/pdf/sajae/v42n2/06.pdf

Nettle, R., Crawford, A., \& Brightling, P. (2018). How private-sector farm advisors change their practices: An Australian case study. Journal of Rural Studies, 58, 20-27. http://dx.doi. org/10.1016/j.jrurstud.2017.12.027

Pinazo, M., \& Bautista-Vallejo, J. (2017). Uso del método bietápico en el estudio de los procesos de enseñanza y aprendizaje musical a través de Moodle. Revista Internacional de Investigación en Ciencias Sociales, 13(2), 187-200. http://dx.doi.org/10.18004/riics.2017.diciembre.187-200

Rendón-Medel, R., Díaz-José, J., Hernández-Hernández, B., \& Camacho-Villa, T. (2015). Modelos de intermediación en la extensión agrícola. Revista Mexicana de Ciencias Agrícolas, 6(1), 139-150. Recuperado el 23 de mayo de 2020, de http://www.scielo.org.mx/pdf/remexca/ v6n1/v6n1a12.pdf 
Rodríguez Espinosa, H., \& Ramírez Gómez, C. (2015). Abordaje metodológico para formulación participativa de planes de asistencia técnica agropecuaria con enfoque territorial. Acta Agronomica, 64(4), 321-329. http://dx.doi.org/10.15446/acag.v64n4.45162

Rosset, P., Machín Sosa, B., Roque Jaime, A., \& Ávila Lozano, D. (2011). The Campesino-toCampesino agroecology movement of ANAP in Cuba: social process methodology in the construction of sustainable peasant agriculture and food sovereignty. The Journal of Peasant Studies, 38(1), 161-191. http://dx.doi.org/10.1080/03066150.2010.538584

Rubio-Hurtado, M., \& Vilà-Baños, R. (2017). El análisis de conglomerados bietápico o en dos fases con SPSS. REIRE. Revista d'Innovació i Recerca en Educació, 10(1), 118-126. http:// dx.doi.org/10.1344/reire2017.10.11017

Sanga, C., Kalungwizi, V., \& Msuya, C. (2013). Building agricultural extension services system supported by ICTs in Tanzania: Progress made, challenges remain. International Journal of Education and Development Using ICT, 9(1), 80-99. Recuperado el 23 de mayo de 2020, de https://files.eric.ed.gov/fulltext/EJ1071386.pdf

Vilà-Baños, R., Rubio-Hurtado, M., Berlanga-Silvente, V., \& Torrado-Fonseca, M. (2014). Cómo aplicar un cluster jerárquico en SPSS. REIRE. Revista d'Innovació $i$ Recerca en Educació, 7(1), 113-127. http://dx.doi.org/10.1344/reire2014.7.1717

Wellard, K., Rafanomezana, J., Nyirenda, M., Okotel, M., \& Subbey, V. (2013). A review of community extension approaches to innovation for improved livelihoods in Ghana, Uganda and Malawi. Journal of Agricultural Education and Extension, 19(1), 21-35. http://dx.doi.or g/10.1080/1389224X.2012.714712 